\title{
Michael Tsokos, Forensic pathology reviews-vol. 5
}

\author{
Totowa, NJ (Humana Press) 2008-292 pages ISBN 978-1-58829-832-4
}

\author{
S. Pollak
}

Received: 2 February 2009 /Accepted: 23 February 2009/Published online: 11 March 2009

(C) Springer-Verlag 2009

The fifth volume of the "Forensic Pathology Reviews" series, which was founded in 2004, has just appeared which shows that its concept and contents have found wide acceptance in the scientific community. The editor, Professor Michael Tsokos, was appointed Director of the Institute of Legal Medicine, Charité-Universitätsmedizin Berlin, in 2007 and is also the Head of the Berlin State Institute of Forensic and Social Medicine. Once again, he has succeeded in obtaining contributions from internationally renowned experts.

The 14 articles of the new volume have been written by authors from Great Britain, Australia, New Zealand, the USA, Singapore, and Germany. In this review, only the titles of the individual chapters can be mentioned: Death due to hypothermia (B. Madea et al.), Fatal falls from a height (E.E. Türk), Biomechanical aspects of craniofacial blunt force injury (J. Kieser et al.), Electrocution and the autopsy (R. Wick and W. Byard), Central nervous system alterations in alcohol abuse (A. Büttner and S. Weis), Deaths following delirium (R. Gill), The role of myocardial bridging in sudden cardiac death (M.J.P. Biggs et al.), Nontraumatic intramuscular haemorrhages in deaths from a natural cause (F. Schulz et al.), Forensic wound ballistics (B. Karger), Identification of unknown victims at autopsy (E.K. Simpson and R.W. Byard), Forensic implications of tattooing (G.E. Cains and R.W. Byard), The roles of the FBI profiler and the forensic pathologist in the investigation of serial murder (T.S. Corey et al.), Forensic histopathology (G. Lau and L.S. Hui), and Age estimation of live adolescents and young adults (A. Schmeling et al.).

As in the preceding volumes of this series, the contributions are all written in English. At the beginning of each text, a concise abstract is given and detailed references at the end provide useful information for further reading on the subject. The articles dealing with morphological aspects comprise numerous instructive illustrations. As the other books of the series, volume 5 of "Forensic Pathology Reviews" succeeds again to present the latest developments in important current fields of classical forensic medicine. The new publication can be recommended without any reservations to all scientists and professionals working in this field.

Stefan Pollak

S. Pollak $(\bowtie)$

Institute of Legal Medicine, University of Freiburg,

Albertstrasse 9,

79104 Freiburg, Germany

e-mail: stefan.pollak@uniklinik-freiburg.de 